\title{
Clinical Implications of Thermoplastic Mask Immobilization on Acute Effects of Radiotherapy in Head and Neck Cancers
}

\author{
Amit Bahl, Sushmita Ghosal, Rakesh Kapoor, Tapesh Bhattacharya, Suresh C Sharma
}

\begin{abstract}
Aim: Thermoplastic immobilization devices are used in head and neck radiotherapy to increase precision of treatment delivery. However, there is concern about increase in radiation induced skin morbidity with the use of such devices. This study was conducted with an aim to evaluate any increase in clinically observed skin reactions with the use of thermoplastic immobilization devices.
\end{abstract}

Materials and methods: Fifty patients of head and neck cancers undergoing radical radiotherapy were randomized into two study groups in this prospective randomized study. Group 'A' received treatment with thermoplastic immobilization device. Group'B' received treatment without any immobilization device. A weekly assessment of normal tissue reactions was done using Radiation Therapy Oncology Group (RTOG) acute radiation acute radiation morbidity scoring criteria.

Results: Increase in skin reactions was seen in patients using thermoplastic devices. Grade 3 reactions were seen in 56 vs $52 \%$ in the study and control group respectively. The difference was however not statistically significant $(p=0.09)$.

Conclusion: Increase in skin dose with use of thermoplastic immobilization devices may not clinically translate into a significant increase in acute skin reactions. Thermoplastic immobilization devices can be safely used in head and neck cancers to ensure accurate radiation delivery.

Keywords: Radiotherapy, Head and neck, Cancer, Immobilization, Skin toxicity.

How to cite this article: Bahl A, Ghosal S, Kapoor R, Bhattacharya T, Sharma SC. Clinical Implications of Thermoplastic Mask Immobilization on Acute Effects of Radiotherapy in Head and Neck Cancers. J Postgrad Med Edu Res 2012;46(4):187-189.

Source of support: Nil

Conflict of interest: None declared

\section{INTRODUCTION}

Head and neck malignancies as a group constitute the most common cancer in the Indian population. ${ }^{1}$ Radiation therapy forms an integral part in both the definitive and adjuvant treatment of various head and neck tumors. Normal tissue toxicity in the form of skin reactions and mucositis are seen in patients undergoing head and neck irradiation. All patients on radiotherapy experience some form of skin toxicity ranging from grade 1 to 4 . The incidence of grade 3 and 4 mucositis may range from 25 to $40 \%$ in patients treated with radiotherapy alone. This increases to 70 to $80 \%$ in concurrent chemoradiotherapy protocols. ${ }^{2}$ Thermoplastic devices have been used for patient immobilization to increase the accuracy of radiation delivery. In our department we use thermoplastic masks (Orifit Industries, Belgium) in all our radically planned head and neck cases. Other devices in use include the polystyrene bead vaccum cradles, carbon fiber support plates. There has been a concern regarding the increase in skin doses as a consequence of bolus effect due to the use of such devices thereby interfering with skin sparing effect of megavoltage radiation. ${ }^{3,4}$ An increase in skin surface dose from 16 to $27 \%$ for $6 \mathrm{MV}$ megavoltage radiation has been documented with use of such immobilization devices. ${ }^{5}$ This may increase the skin toxicity and can be detrimental for the patient's quality of life. However, any increase in skin toxicity must be viewed keeping in mind the increased precision in radiotherapy delivery which is achieved by the use of these devices. We report here the acute toxicity seen with the use of thermoplastic immobilization devices in head and neck cancers patients.

\section{MATERIALS AND METHODS}

Fifty patients of head and neck cancers undergoing radical radiotherapy were randomized into two study groups. Randomization was done using computer generated randomization tables. The inclusion criteria included:

- Histologically proven squamous cell carcinoma of head and neck

- Karnofsky performance status $>60$

- Patients with stage I, II and III disease

- Patients planned for definitive radiotherapy.

Exclusion criteria included:

- Age more than 70 years

- Patients receiving concurrent chemoradiotherapy

- Any prior radiotherapy treatment.

The study group (group A) included 25 patients receiving radical radiotherapy with a thermoplastic head and neck immobilization mask (Orifit Industries Belgium). The thermoplastic mask used had a thickness of $2 \mathrm{~mm}$ and density of $1.13 \mathrm{gm} / \mathrm{cc}$. The control group (group B) included 25 patients on radical radiotherapy without thermoplastic head and neck immobilization device.

Radical radiotherapy was delivered to a total dose of 70 Gray over 35 fractions over a 7-week period. Bilateral 
parallel opposed face and neck portals with shrinking field technique were used for the treatment. Spinal cord shielding was done at a dose of 44 Gray. Compensators were made for all patients. All patients were treated on a cobalt 60 treatment unit (Theratronics 780E).

Radiotherapy toxicity was graded as per radiation therapy oncology group (RTOG) toxicity criteria at weekly intervals for the entire duration of treatment. Patients in both groups were given similar instructions on precautions to be practiced during radiotherapy treatment and maintaining good oral hygiene.Chlorhexidine mouth washes were used by all patients on treatment.

For statistical analysis data was arranged in Excel format and converted to SPSS format. To see the significance association Chi-square test has been performed. To see trend within a group we did a two-way ANOVA with appropriate post hoc analysis. A p-value of less than 0.05 was considered as statistical significant.

\section{RESULTS}

Fifty patients were available for final evaluation. There were 38 male and 12 female patients. The median age of the patients was 48 years. The various subsites of head and neck cancers evaluated included carcinomas of base of tongue (32\%), tonsil (24\%), nasopharynx (12\%), pyriform sinus (8\%), anterior tongue (6\%), secondaries neck with unknown primary (12\%) and buccal mucosa (6\%). Six patients had stage 1 disease, 36 had stage 2 and eight patients had stage 3 disease. The results are given in Table 1 .

Skin reactions: Grade 2 skin reaction was seen in 10 patients (40\%) using thermoplastic immobilization devices while grade 3 reaction was seen in 14 patients (56\%). One patient developed grade 4 reaction. In the control group 10 patients (40\%) patients had grade 2 skin reactions. Grade 3 reaction was seen in 52\% patients. Two patients developed grade 4 reaction. Though a trend toward higher grade 3 skin reaction was seen in patients using thermoplastic masks, the difference was not statistically significant $(\mathrm{p}=0.09)$.

Salivary gland: Grade 2 salivary gland toxicity was seen in $68 \%$ of patients in the study group. The control group had a $64 \%$ incidence of grade 2 toxicity. The difference was not statistically significant.
Pharynx and esophagus: A similar incidence of grade 1 and 2 toxicity was seen in the two treatment groups. While 18 patients (72 \%) of patients using mask immobilization had grade 1 and 2 reactions, grade 3 reactions were seen in $28 \%$ in both treatment arms.

Larynx: An equal incidence of larangeal reactions was seen on the two treatment groups. Among $60 \%$ of patients on both treatment arms showed grade 1 and 2 reactions.

Mucositis: A comparable incidence of mucositis was seen on the two treatment groups. Among patients using mask immobilization $88 \%$ had grade 2 and 3 reactions. Among $80 \%$ patients in the control group exhibited similar reactions.

Acceptability: All patients using orifit were questioned about their acceptability and comfort level with using thermoplastic immobilization devices. None of the patients experienced any claustrophobia nor had difficulty in using the thermoplastic immobilization masks.

\section{DISCUSSION}

Conventional radiation therapy treatment in head and neck region poses problems related to accurate daily repositioning of patient. This is important in definitive radiotherapy courses where treatment is usually delivered over a 7 weeks period. Patient movement during treatment can lead to delivery of unintended dose to surrounding critical structures like eyes, brain, brainstem, etc. Accurate repositioning of patient on a daily basis forms one of the most important steps in the entire treatment delivery process. This is of particular significance in younger patients with early stage disease where long-term survival is expected and long-term morbidity is of consequence.

Thermoplastic immobilization devices play a crucial role in ensuring accurate delivery of radiation and minimizing day to day set-up variation. Concerns expressed regarding increase in surface dose due to these devices may be relevant, only if the increase in skin dose leads to a clinically appreciable increase in acute toxicity and long-term radiation induced changes. Halm et al studying thermoplastic devices demonstrated an increase in cobalt 60 gamma-rays dose from 57.1 to $77.7 \%$ for $0.5 \mathrm{~mm}$ water depth and an increase in $6 \mathrm{MV}$ X-ray from 49.5 to $63.2 \%$ for $0.5 \mathrm{~mm}$ water depth. ${ }^{6}$ Electron beam of $10 \mathrm{MeV}$ showed an increase from 82.2 to $86.1 \%$ for $0.5 \mathrm{~mm}$ water depth

\begin{tabular}{|c|c|c|c|c|c|c|c|c|}
\hline & \multicolumn{4}{|c|}{ Group A (\%) } & \multicolumn{4}{|c|}{ Group B (\%) } \\
\hline & Grade 1 & Grade 2 & Grade 3 & Grade 4 & Grade 1 & Grade 2 & Grade 3 & Grade 4 \\
\hline Skin & - & 40 & 56 & 4 & - & 40 & 52 & 8 \\
\hline Salivary gland & 32 & 68 & - & - & 36 & 64 & - & - \\
\hline Pharynx and esophagus & 72 & - & 28 & - & 72 & 28 & - & - \\
\hline
\end{tabular}


with the use of thermoplastic masks. However, in vivo dosimetry during treatment showed these doses to be within $\pm 5 \%$ of the prescribed doses in $92.3 \%$ of cases. Carl et al have showed that increase in skin dose using parallel opposed treatment fields shows a relationship with the thickness of the material in $\mathrm{mg} / \mathrm{cm}$. With thermoplastic materials with thickness less than $100 \mathrm{mg} / \mathrm{cm}$ the skin doses obtained are comparable with those produced by variation in source to skin distance, shielding trays and field size. ${ }^{7}$ Use of smaller head mask has shown to further reduce skin reactions compared to head and shoulder masks. ${ }^{8}$ For patients experiencing claustrophobia Kim et al have suggested the use of Vac Fix mold. ${ }^{9}$ Thermoplastic sheets used for immobilization usually have holes in them to reduce the dose buildup effect. In a recent analysis on use of thermoplastic masks in patients with larangeal cancers Peng et al concluded that with having opening window on the mask considerably reduces the risk of radiodermatitis. ${ }^{10}$ Though our results show a trend toward increase in skin reactions the results are not statistically significant. In our study the incidence of grade 3 skin reactions was 56 vs 52\% for the study and the control group respectively. A comparable incidence of mucositis was seen between the study arms. An incidence of grade 3 and 4 mucositis of 25 to $40 \%$ is reported in literature. ${ }^{11}$ In our patients we did not encounter the problem of claustrophobia. Adequate patient awareness regarding the purpose of immobilization helps in increasing compliance during treatment. Maintenance of good oral hygiene practices may further help to keep radiation induced morbidity within acceptable limits.

\section{CONCLUSION}

Increase in skin dose with use of thermoplastic immobilization devices may not clinically translate to a significant increase in acute skin reactions. Long-term effects however need to be studied. Thermoplastic immobilization devices can be safely used in head and neck cancers to ensure accurate radiation delivery.

\section{REFERENCES}

1. National cancer registry programme (1981-2001). An overview. Indian Council of Medical Research 2002.

2. Fu K, Pajak T, Trotti A. A radiation therapy oncology group (RTOG) phase III randomized study to compare hyperfractionation and two variants of accelerated fractionation to standard fractionation radiotherapy for head and neck squamous cell carcinomas. First report of RTOG 9003. Int J Radiat Oncol B Phys 2000;48:7-16.
3. Mellenberg DE. Dose behind various immobilization and beammodifying devices. Int J Radiat Oncol Biol Phys 1995;15:32(4): 1193-97.

4. Fiorino C, Cattaneo GM, del Vecchio A, et al. Skin dose measurements for head and neck radiotherapy. Med Phys 1992;19(5):1263-66.

5. Hadley SW, Kelly R, Lam K. Effects of immobilization mask material on surface dose. J Appl Clin Med Phy 2005;6(1):1-7.

6. Halm EA, Tamri A, Bridier A, Wibault P, Eschwege F. Influence of thermoplastic mask on the absorbed skin dose for head and neck tumor radiotherapy. Cancer Radiother 2002; 6(5):310-19.

7. Carl J, Vestergaard A. Skin damage probabilities using fixation materials in high energy photon beams. Radiother Oncol 2000;55(2):191-98.

8. Sharp L, Lewin F, Johansson H, Payne D, Gerhardsson A, Rutqvist LE Randomized trial of two types of thermoplastic mask for patient immobilization during radiation therapy for head and neck cancer. Int J Radiat Oncol Biol Phys 2005;1: 61;(1):250-56.

9. Kim S, Akpati HC, Li JG, Liu CR, Amdur RJ, Palta JR. An immobilization system for claustrophobic patients in head and neck intensity modulated radiation therapy. Int J Radiat Oncol Biol Phys 2004;(1);59(5):1531-39.

10. Peng G, Chen J, Cao R, et al. Study on influence of thermoplastic mask on radiation dermatitis for patients with laryngeal cancer. Lin Chung Er Bi Yan Hou Tou Jing Wai Ke Za Zhi 2011;25: 1120-22.

11. Adam SG. Mucositis: Current management and investigations. Sem Rad Oncol 2003;13(3):267-73.

\section{ABOUT THE AUTHORS}

\section{Amit Bahl}

Assistant Professor, Department of Radiotherapy, Postgraduate Institute of Medical Education and Research, Chandigarh, India

\section{Sushmita Ghosal}

Professor, Department of Radiotherapy, Postgraduate Institute of Medical Education and Research, Chandigarh, India

\section{Rakesh Kapoor (Corresponding Author)}

Additional Professor, Department of Radiotherapy, Postgraduate Institute of Medical Education and Research, Chandigarh, India Phone: 987-264-8344, e-mail: drkapoor.r@gmail.com

\section{Tapesh Bhattacharya}

Senior Resident, Department of Radiotherapy, Postgraduate Institute of Medical Education and Research, Chandigarh, India

\section{Suresh C Sharma}

Professor and Head, Department of Radiotherapy, Postgraduate Institute of Medical Education and Research, Chandigarh, India 